\title{
RINDIK VOICE SYNTHESIS USING MODIFIED FREQUENCY MODULATION AS BALI CULTURAL PRESERVATION EFFORTS
}

\author{
${ }^{\mathrm{a} I}$ Made Widiartha, ${ }^{\mathrm{b}}$ Agus Muliantara \\ ${ }^{a, b}$ Informatics Engineering Program, Department of Computer Science \\ Faculty of Mathematics and Natural Sciences, Udayana University \\ E-Mail: imadewidiartha@cs.unud.ac.id
}

\begin{abstract}
Gamelan bali is one aspect of the art highly favored by both domestik and foreign tourists. One type of popular typical Balinese gamelan is Rindik. Rindik is one of Balinese traditional musical instrument made of bamboo. Now days, the number of foreign culture and today's lifestyle give some impacts on the declining interest of Balinese to interact with this type of conventional gamelan. The younger generation is now more inclined to like devices which are played through electronics/software component. To increase public interest towards traditional gamelan bali especially rindik we need a breakthrough to digitize gamelan rindik and presenting it in the form of rindik software application. Today's advanced technology has made a way to digitize a wide range of instruments including rindik into computerized form. For example we can use frequency modulation as a voice synthesis technique. This method was developed by researchers in the field of sound synthesis. In this research, we have done the sound synthesis process of rindik instrument into digital form using frequency modulation. The best results were obtained through the synthesis comparison of carrier signal frequency and modulator is 1:7. Outcomes of this research is a digitizing result which is presented in the form of a gamelan rindik package on desktop based software application.
\end{abstract}

Keyword: gamelan rindik, voice synthesis, frequency modulation 


\section{INTRODUCTION}

Bali province is one of the areas in the Indonesia archipelago that is famous for its wealth of art and culture. Art and culture are two main things in Balinese daily life. The pattern of life has spawned a variety of things such as musical instruments, dances, traditional clothes, traditional houses, handicrafts, etc. This leads to increasingly more tourists come to Bali to enjoy the arts and culture of Bali.

One aspect of Balinese art favored by tourists is typical Balinese gamelan. This is due to the unique of dynamics and strong ethnic imagery. One type of popular typical Balinese gamelan is rindik. Rindik is one of Balinese traditional musical instrument made of bamboo. It consists of eleven bamboos which formed in different size so that it can produce a harmonious tone. Over time, the number of foreign culture that goes to bali and lifestyle of today's advanced technology era has had an impact on the declining interest of young people of bali to interact with this type of conventional gamelan. Today's young generation is more interested in the technology equipment as well as modern instruments in the form of electronic devices and software.

To preserve and increase public interest towards Balinese traditional gamelan especially rindik, we need a breakthrough to digitize gamelan rindik and present it in the form of electronic devices. It will be a bridge to the younger generation in order to maintain the interest and appeal to traditional gamelan especially rindik. The era of today's advanced technology has made a way for us to digitize a wide range of instruments including rindik into computerized form. In the field of sound processing, there is a technique called synthesis. Synthesis is a technique used to generate specific sound into digital form.

Until now, efforts have been made and many studies of researchers try to develop a digital music by voice synthesis. In previous studies, it has been used an Analysis model of Synthesis to the Javanese gamelan [1]. This model has not been able to produce a distinctive clatter of metal. Renda Eko Saputra and his colleagues were also conducting research with the title Analysis and Synthesis of Sound Strings In Semi-Acoustic Guitar [2]. In his study Eko Renda using additive synthesis method where the results already quite close to or equal to the sound produced by the guitar. Other research to synthesize the sounds of brass, woodwind, and percussion sounds using the average method Frequency Modulation (FM) [3].From research conducted by Pablo Caceres to the topic Sound Design Learning the result is that this FM synthesis method with the algorithm that is fast and able to harmonize various tonal variations [4].

In another research, the document direct synthesis versus wavetable synthesis said that the direct synthesis (FM included in it) has the advantages of consuming storage space [5]. It is less than other methods, it also has an excellent sound accuracy by utilizing mathematical formulation, and it is very good to a sound that has many variations. Having regard to the performance of the FM method, so that this research will implement FM method for synthesizing voice of gamelan rindik.

\section{LITERATURE REVIEW}

\section{Gamelan Rindik}

Gamelan Rindik is one of the traditional musical instrument originating from the island of Bali. Rindik made of bamboo which consist of several sticks of bamboo. Each stick of bamboo has different size one to another. Rindik composed of 11-14 bamboo stems are cut and then arranged on the left of the larger size to a smaller size on the right. Bamboo poles are arranged in a row, tied, and framed with wood which has been decorated with carved and painted.

Rindik played with two long hitter instrument made of bamboo rods that has been refined and given a rubber dots on the ends. Rubber is hitting the bamboo on rindik so as to produce a harmonious sound. Rindik organized into two types of tones, they are lanang (rhythm of a song, produce bass, bamboo is longer and large, located sequentially from a low pitch to high, from left to right) and wadon (melodic of a song, produce a sound high, bamboo shorter and smaller size, located sequentially from low to high tones after lanang). Tones produced by rindik are compiled base on selendro tone system. Selendro tone system is tone system known in 
Bali as "Saih Lima" which only consists of five types of tones these are nding, ndong, ndeng, ndung, ndang, and a high nding tone.

Rindik is usually played by two players, where one acts as "penyangsih" which plays a different tone and tempo to the other player that produce sound blared. The rindik musical instruments require coordination between right and left hands where right and left hand hold different tasks in each song. The left hand plays a fundamental tone of a song that is part of "lanang" from rindik, and the right hand plays the melody of the song that is part of "wadon" of rindik so that the left and right hand coordination plays an important role in producing beautiful song. The sound of rindik are natural and relax. Therefore, it is often played as a greeting at places like airports, hotels and civil ceremonies in Bali.

\section{Basic Frequency and Frequency of Harmony}

\section{Keep in mind that a signal is not a sine wave.}
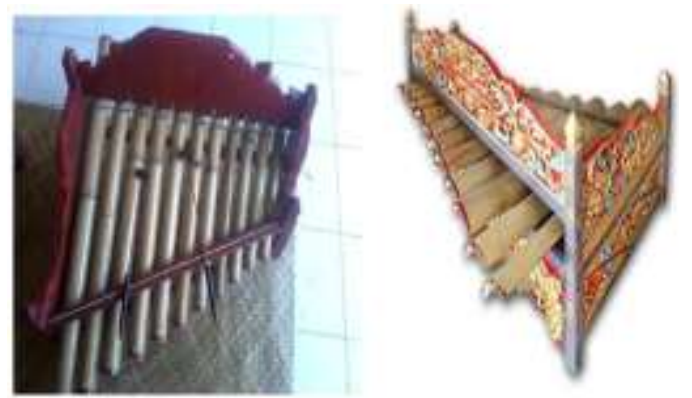

Figure 1. Gamelan Rindik

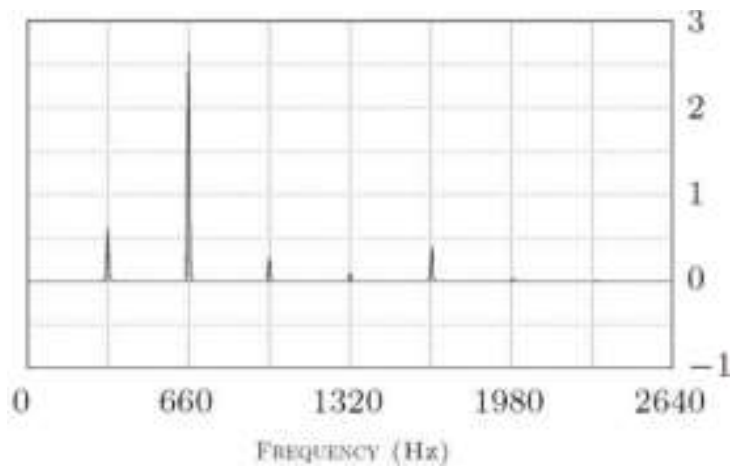

Figure 1 : Time Domain of Piano Note E4 Voice Signal
The original signal has a range of frequencies, different amplitude, and disorders. A signal has two frequencies, namely the fundamental frequency and harmonic frequency.

The fundamental frequency is the lowest frequency of a periodic signal wave. While the frequency of harmony is the frequency components of the signal which are multiples of the fundamental frequency [6]. As an example of the fundamental frequency and the harmonic frequency of a signal is shown in Figure 2.

From Figure 2 there is a peak at a frequency of $330 \mathrm{~Hz}, 660 \mathrm{~Hz}, 990 \mathrm{~Hz}, 1320 \mathrm{~Hz}$, and 1620 Hz. It can be seen that these values are multiples of the frequency of the first peak, which is $330 \mathrm{~Hz} .330 \mathrm{~Hz}$ frequency is called the fundamental frequency and at the same time as the first harmony. For frequency $660 \mathrm{~Hz}$ is the second harmony. It is the first overtone of the fundamental frequency. Overtone is a frequency which is higher than the fundamental frequency and a multiples value of the fundamental frequency value.

\section{Cent}

Cent is a logarithmic unit of measure used for musical intervals. This measuring technique proposed by Alexander J.Ellis (Ellis, 1885). As decibels relation to the intensity, cent is the ratio between the two close frequencies. If known frequencies $a$ and $b$ of the two tones, values cent (n) to measure from $a$ to $b$ can be calculated by using the Equation (1).

$$
n=200 . \log _{2}\left(\frac{b}{a}\right)
$$

\section{Hilbert Transformation}

Hilbert transformation is a powerful technique which can be used to look for the envelope of a signal [7]. The formula of Hilbert transform can be seen in Equation (2).

$$
x_{H}(t)=\frac{1}{\pi t} x(t)
$$

Where $\boldsymbol{x}_{\boldsymbol{H}}$ is the Hilbert transform result signal, $\mathrm{x}$ original sound signal, and $\mathrm{t}$ is the time. 


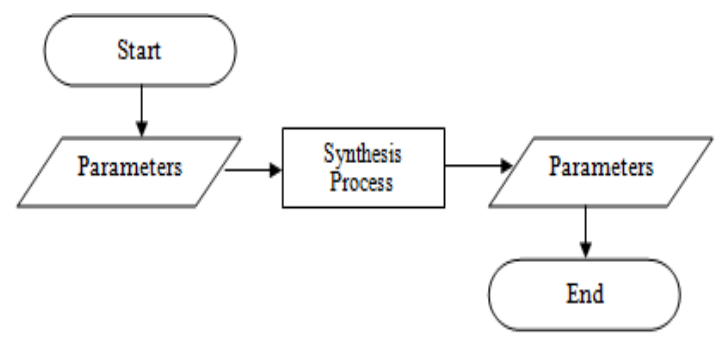

Figure 2 : Flow Sound Synthesis Process in General

\section{Synthesis}

Synthesis is a strategy or technique to generate a sound by using a parameter to control raised voice. Figure 3 shows the general process of sound synthesis techniques. The parameters used are the packaging signal and the fundamental frequency of a sound which will be. The resulting sound is the synthesized sound based on the parameters that have been determined and are heard.

\section{Modified Frequency Modulation (ModFM)}

ModFM is an improved method of Frequency Modulation method. ModFM is stated by Victor Lazzarini and Joseph Timoney in 2010 [8]. FM method is modified by normalizing the Bessel function and produce ModFM equation shown in Equation (3).

$$
s(t)=\frac{A}{e^{k}} \sum_{n=-\infty}^{\infty} I_{n}(k) \cos \left(\omega_{c} t+n \omega_{m} t\right)
$$

Note :

$$
\begin{aligned}
& s(t): \text { the signal that has been } \\
& \text { modulated } \\
& \begin{array}{l}
A: \text { envelope signal } \\
I \quad: \text { Normalized } \\
\quad\left(\mathbf{I}_{\mathbf{n}}(\mathbf{k}) \approx \frac{\mathrm{e}^{\mathbf{k}}}{\sqrt{2 \pi \mathbf{k}}}\right)
\end{array}
\end{aligned}
$$

$k$ : modulation index

\section{FLOW OF SYNTHESIS PROCESS}

Figure 4 shows the process flow of the ModFM method. On the chart is seen that input in this system is a rindik recorded sound which has been obtained previously. The first stage of the process is to analyze the fundamental frequency of the sound recordings using Sound Forge program.

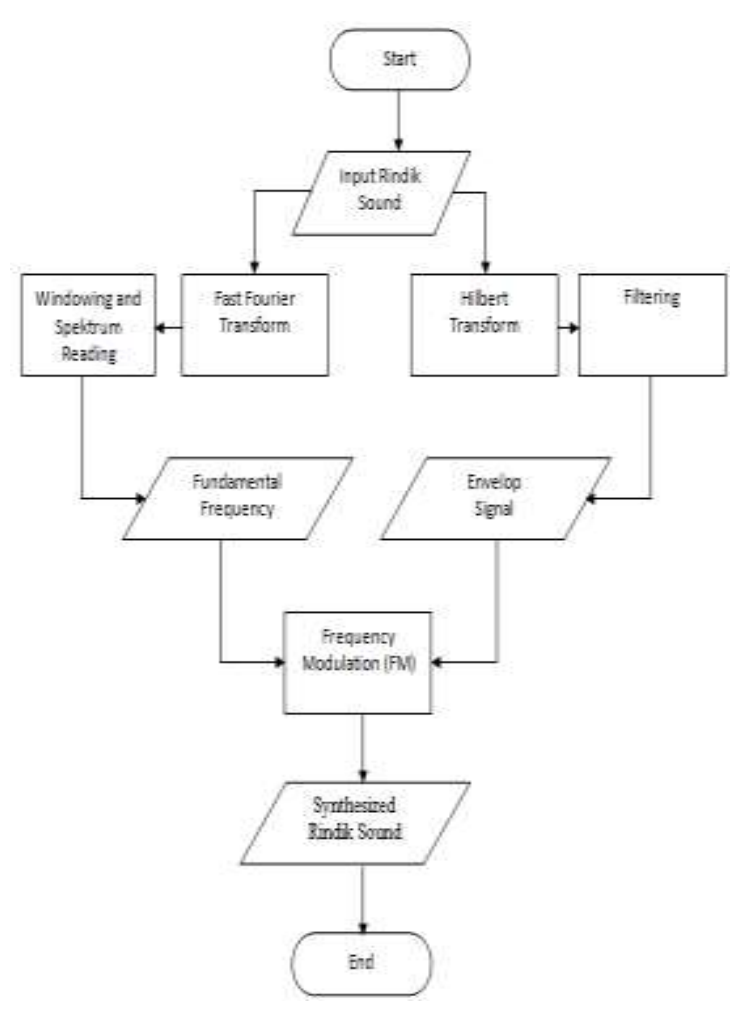

Figure 4: Flow of ModFM Method

Then proceed envelope signal search using Hilbert transformation and a moving average filter. The last step is a process of synthesis using methods ModFM.

\section{RESULTS AND DISCUSSION}

\section{Basic frequency of Gamelan Rindik}

To get the range of the rindik fundamental frequency, data was collected as many as 120 voice rindik from six different rindik which consist of eleven bars (bamboo). From the initial processing using Sound Forge 10.0 program obtained the highest frequency for each bar. With the approach of overtone, the fundamental frequency of the gamelan rindik lies in the frequency range as shown in Table 1 and Table 2.

The average value of fundamental frequency of rindik bar is used as the input on the synthesis process. The result of time domain analysis using the program Sound Forge 10 obtained the highest frequency of all 11 sound bars are shown in Table 2. 


\section{Input of Synthesis Process}

In addition to the average fundamental frequency and the highest in Table 1 and Table 2 , other input of synthesis process is the ratio of the carrier signal frequency and modulator.

The values of comparison are combination between the carrier signal value and modulation signal value. These are the value between 1 to 9 (integer number). Another input of synthesis process is envelope signal. Envelope signal which is used in this synthesis process is a signal envelope of the dataset that has the lowest fundamental frequency difference with the average frequency for each bar. Selected envelopes are shown in Table 3.

Table 1. Distance and Average Fundamental Frequency of Rindik

\begin{tabular}{cccc}
\hline Bar & Min $(\mathrm{Hz})$ & Max $(\mathrm{Hz})$ & Average \\
\hline 1 & 19,4 & 21,8 & 20,6 \\
2 & 21,4 & 24,5 & 22,9 \\
3 & 26 & 30 & 28,3 \\
4 & 29,25 & 33,1 & 31,3 \\
5 & 32,75 & 37,1 & 34,8 \\
6 & 39,5 & 45,3 & 42,2 \\
7 & 43,9 & 50,6 & 46,8 \\
8 & 52,9 & 62,5 & 57,1 \\
9 & 59,25 & 67,1 & 62,9 \\
10 & 66,4 & 74,8 & 70.1 \\
11 & 79,6 & 93,6 & 85,4
\end{tabular}

Table 2. Range and Average Highest Frequency Gamelan Rindik

\begin{tabular}{cccc}
\hline Bar & Min $(\mathrm{Hz})$ & Max $(\mathrm{Hz})$ & Average \\
\hline 1 & 155 & 174 & 165,2 \\
2 & 171 & 196 & 182,8 \\
3 & 208 & 240 & 226,2 \\
4 & 234 & 265 & 250,2 \\
5 & 262 & 297 & 278,7 \\
6 & 316 & 362 & 337,3 \\
7 & 351 & 405 & 374,7 \\
8 & 423 & 500 & 456,5 \\
9 & 474 & 537 & 503 \\
10 & 531 & 598 & 561,7
\end{tabular}

\begin{tabular}{cccc}
\hline Bar & Min $(\mathrm{Hz})$ & Max $(\mathrm{Hz})$ & Average \\
\hline 11 & 637 & 749 & 683 \\
\hline
\end{tabular}

Table 3. Dataset for the extraction of the signal envelope

\begin{tabular}{llllllllllll}
\hline Bilah & 1 & 2 & 3 & 4 & 5 & 6 & 7 & 8 & 9 & 10 & $\begin{array}{l}1 \\
1\end{array}$ \\
\hline $\begin{array}{l}\text { Datase } \\
\mathrm{t}\end{array}$ & 4 & 4 & 6 & 6 & 4 & 4 & 4 & 4 & 4 & 4 & 4 \\
\hline
\end{tabular}

\section{RESULT AND DISCUSSION}

There are two scenarios in this phase. The first and second experiment was performed using all bars in gamelan rindik. The first scenario by calculating the average difference between the dataset harmonic frequencies with speech synthesis products. The second scenario is to look whether the synthesis product fundamental frequency is at tolerance value or not. Table 4 shows the average difference in the harmony frequency for the entire experiment. From the results, the best combination between carrier signal frequency and modulator obtained is 1:7 for all bar.

The fundamental frequency which is generated from the best combination is shown in Table 5. The highest frequency value generated from the best combination is shown in Table 6.

From the table it can be observed that the value of the fundamental frequency of the voice synthesis product with the best ratio was in the range shown in Table 1 and Table 2.

Tabel 4. Average Difference Frequency Synthesized Harmony with The Best Combination

\begin{tabular}{cc}
\hline Bar & value Difference \\
\hline 1 & 6 \\
2 & 0 \\
3 & 7 \\
4 & 18 \\
5 & 10 \\
6 & 0 \\
7 & 1 \\
8 & 17 \\
9 & 9 \\
10 & 24
\end{tabular}




\begin{tabular}{cc}
\hline Bar & value Difference \\
\hline 11 & 15 \\
\hline
\end{tabular}

Tabel 5. The Fundamental Frequency Synthesized Voice Rindik

\begin{tabular}{cc}
\hline Bar & Fundamental frequency \\
\hline 1 & 20.6 \\
2 & 22.9 \\
3 & 28.3 \\
4 & 31.3 \\
5 & 34.8 \\
6 & 42.1 \\
7 & 46.6 \\
8 & 57.0 \\
9 & 62.9 \\
10 & 70.0 \\
11 & 85.3 \\
\hline
\end{tabular}

Tabel 6. The Highest Frequency Value of Synthesized Voice Rindik

\begin{tabular}{cc}
\hline Bar & Highest Frequency \\
\hline 1 & 165,4 \\
2 & 183,3 \\
3 & 226,1 \\
4 & 250,5 \\
5 & 278,3 \\
6 & 337,2 \\
7 & 373,5 \\
8 & 456,1 \\
9 & 503 \\
10 & 560,3 \\
11 & 682,6 \\
\hline
\end{tabular}

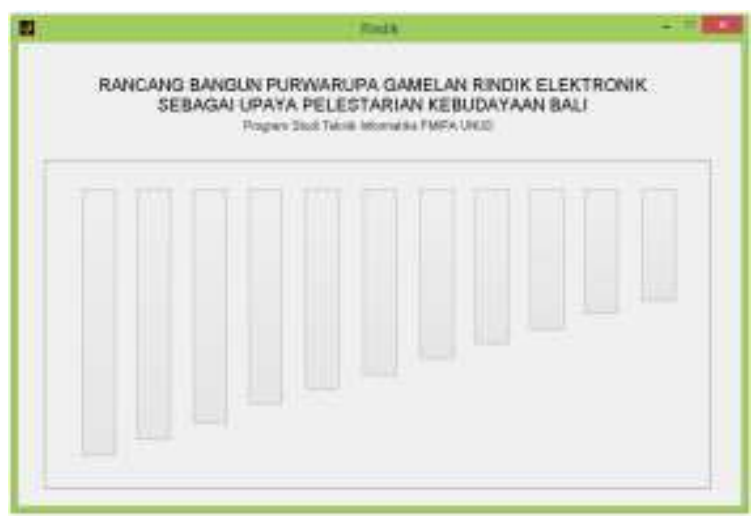

Figure 6 : Comparison Between Highest
Frequency Rindik Dataset and Synthesized Voice
Figure 6 : Comparison Between Highest
Frequency Rindik Dataset and

the result of this synthesis is presented in a user interface.

This research has finished to build a simple desktop based application to play gamelan rindik. This application represents the sound bar into a few buttons. The user interface of the application is shown in Figure 5.

Figure 5 shows the application interface rindik where there are 11 buttons that represent the leaf bar of gamelan rindik.

Applications rindik is made based on the results of research that the best combination of the carrier signal frequency and modulator were obtained for the entire bar is 1: 7. The combination has made synthesized voice of rindik relatively similar to the sound of original voice rindik dataset. The difference frequency harmonies between synthesized voice and original dataset is in the recommended range from 1 to 24. Figure 6, 7, and 8 show a comparison chart between the dataset with the value resulting from the synthesis process.

Figure 6 and 7 show a very little difference between the highest and the fundamental frequency of the sound generated by the synthesis result dataset. It is the same with frequency harmony shown in Figure 8 where the difference is very small.

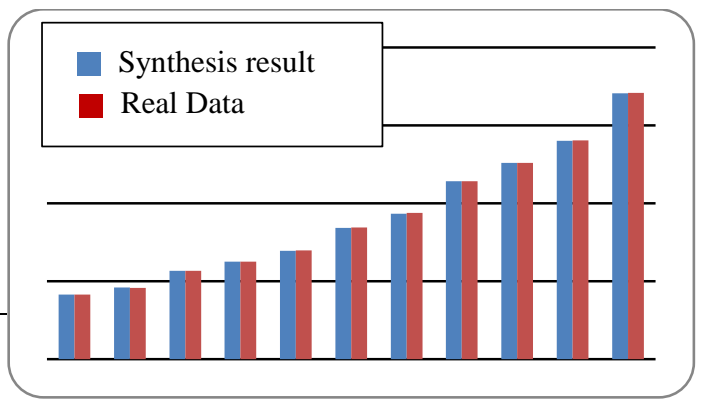

Figure 3. User Interface of Application

After the synthesis process is completed, 


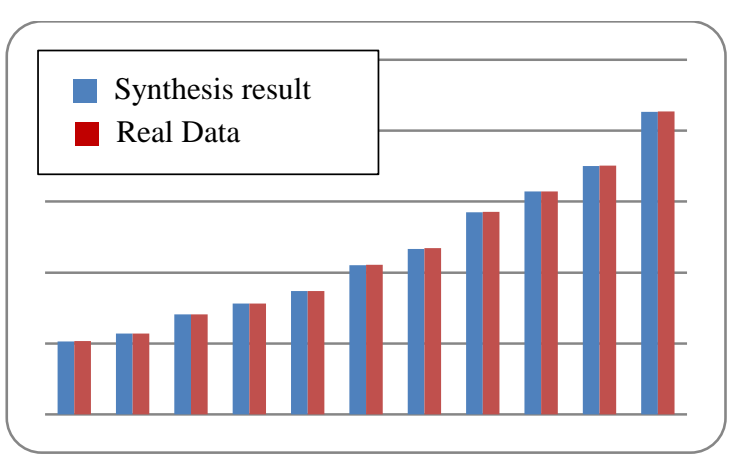

Figure 7 : Comparison Between The Fundamental Frequency Rindik Dataset And Synthesized Voice

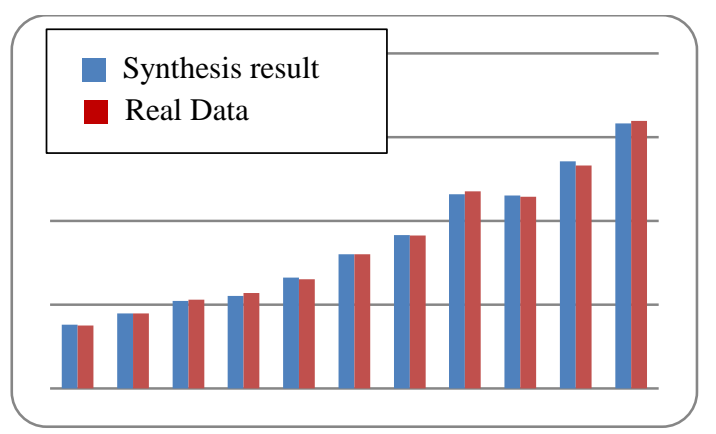

Figure 8: Comparison Between The Frequency of The First Harmony Dataset with Synthesized Voice

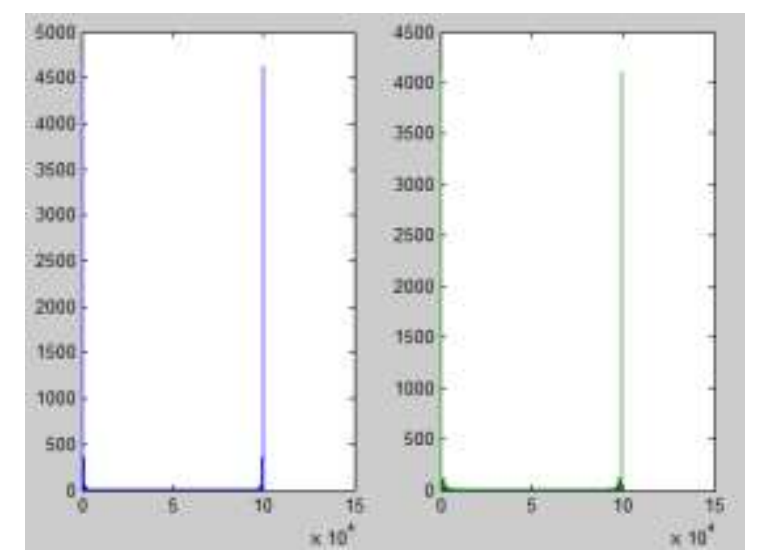

Figure 9: Domain Frequency Synthesized Voice

The synthesized bars which has the smallest average difference of harmony frequency value are the second bar and the sixth bar of gamelan Rindik. The sound produced was fully similar to the original sound. It can be seen from the similarity harmony frequency in figure 8 and fundamental frequency produced (Table 5), which has been in the range allowed (table 1 and table 2) for each gamelan rindik bar.

But the entire voice synthesized voice has a slightly different color from the original sound when heard. The cause of this sound color difference can be seen from the frequency domain of each sound. Figure 9 is a result of the frequency domain of synthesized voice. There are differences in the magnitude frequency. From the results of the frequency domain can also be observed that there are some new harmony frequencies that arise and affect the color of the voice.

\section{CONCLUSION}

From the test results and evaluation of research that has been done, it can be concluded as follows:

1. The synthesis technique using ModFM method has been successfully carried out. The best combination between carrier frequency and modulator is 1:7 where the average difference of harmony frequency between voice synthesis product and original voice is in the range of $1 \mathrm{~Hz}$ to 24 $\mathrm{Hz}$. This is caused by there are differences in the average difference for the fundamental frequency of each bar in the dataset used. The greater the difference is then followed by a large increase in the average difference in the frequency of harmony.

2. Synthesized voice has had the same tone with the sound of the entire dataset as evidenced by the fundamental frequency of the synthesized voice is in the recommended fundamental frequency range of each bar.

\section{REFERENCES}


[1] A. Tjahyanto, Y. K. Suprapto, and D. P. Wulandari, "Model Analysis-By-Synthesis Aplikasi Pembangkit Suara Gamelan Sintetik," Semin. Nas. Apl. Teknol. Inf.

[2] E. R. Saputra, A. Purwanto, and Sumarna, "Analisa dan Sintesa Bunyi Dawai Pada Gitar Semi-Akustik," Semin. Nas. MIPA Yogyak. FMIPA Univ. Negeri Yogyak.

[3] J. M. Chowning, "The synthesis of complex audio spectra by means of frequency modulation," J. Audio Eng. Soc., vol. 21, no. 7, pp. 526-534, 1973.

[4] J.-P. Cáceres, "Sound Design Learning For Frequency Modulation Synthesis Parameters."

[5] P. Burk and C. A. San Rafael, Direct Synthesis versus Wavetable Synthesis. Mobileer, 2004.
[6] J. F. Alm and J. S. Walker, "Timefrequency analysis of musical instruments," Siam Rev., vol. 44, no. 3, pp. 457-476, 2002.

[7] R. L. Allen and D. W. Mills, Signal analysis: time, frequency, scale, and structure. Piscataway, NJ: [Hoboken, N.J.?]: IEEE Press; Wiley-Interscience, 2004.

[8] V. Lazzarini and J. Timoney, "Theory and practice of modified frequency modulation synthesis," J. Audio Eng. Soc., vol. 58, no. 6, pp. 459-471, 2010. 